\title{
Multiple vacua in two-dimensional Yang-Mills theory
}

\author{
A. Bassetto and F. Vian \\ Dipartimento di Fisica "G. Galilei" and INFN, Sezione di Padova, \\ via Marzolo 8, 35131 Padua, Italy \\ L. Griguolo \\ Dipartimento di Fisica "M. Melloni" and INFN, Gruppo Collegato di Parma, \\ viale delle Scienze, 43100 Parma, Italy
}

\begin{abstract}
Two-dimensional $S U(N)$ Yang-Mills theory is endowed with a non-trivial vacuum structure ( $k$-sectors). The presence of $k$-sectors modifies the energy spectrum of the theory and its instanton content, the (Euclidean) space-time being compactified on a sphere. For the exact solution, in the limit in which the sphere is decompactified, a $k$-sector can be mimicked by the presence of $k$-fundamental charges at $\infty$, according to a Witten's suggestion. However, this property neither holds before decompactification nor for the genuine perturbative solution which corresponds to the zero-instanton contribution on the sphere.
\end{abstract}

\section{Introduction}

The vacuum structure in non-abelian quantum gauge theories is still far from being satisfactorily understood. Many important aspects of four-dimensional QCD, such as chiral symmetry breaking, are believed to be related to the existence of multiple vacuum states (labelled by a topological parameter, the $\theta$ angle) and to quantum tunnelling between them mediated by instanton effects. Unfortunately four-dimensional QCD appears too complicated to deal with. Nevertheless, in the Light-Front (LF) formulation of a Quantum Field Theory, where the theory is quantized not on a space-like surface but on a light-like one (see [1] for an extensive review), at least in principle, the exact ground state is described as a simple Fock vacuum. Actually, in four-dimensional gauge theories such an approach is far from being simple, due to the intricate dynamics of the so-called zero-modes. This problem does not exist in two dimensions 
(zero-modes are related to transverse degrees of freedom in LF quantization) and recently it has been shown [2] that, at the perturbative level, LF quantization encodes a complicated instanton dynamics present in the equal-time (ET) formulation. Therefore two-dimensional gauge theories candidate themselves as the simplest models in which the influence of topological parameters on physical quantities can be probed.

\section{$2 \quad k$-sectors and instantons}

The best example of a theory which admits multiple vacua and shares relevant features with four-dimensional gauge theories is $Q C D_{2}$ with adjoint fermions, as noticed many years ago by Witten [3]. What is remarkable is that adjoint matter in some sense mimics transverse degrees of freedom, thus inducing a complex behaviour. We limit ourselves to investigate the $S U(N)$ case when fermionic dynamics is essentially frozen, considering infinitely massive adjoint quarks and studying the static potential between them. Since Yang-Mills fields transform in the adjoint representation, the true local symmetry is the quotient of $S U(N)$ by its center, $Z_{N}$. A standard result in homotopy theory tells us that the quotient is not simply connected, the first homotopy group being $\Pi_{1}\left(S U(N) / Z_{N}\right)=Z_{N}$. This result is of particular relevance for the vacuum structure of a two-dimensional gauge theory: in the case at hand we have exactly $N$ inequivalent quantizations, parametrized by a single integer $k$, taking the values $k=0,1, . ., N-1$. Concerning the pure $S U(N)$ Yang-Mills theory, the explicit solution when $k$-states are taken into account was presented in Ref. [4]: their main result, the heat-kernel propagator on the cylinder, allows to compute partition functions and Wilson loops winding around a smooth non self-intersecting closed contour on the sphere $S^{2}$. Wilson loops, in this case, strongly depend on $k$ and, for the theory defined on the plane, read

$$
\begin{gathered}
\mathcal{W}_{k}(A)=\frac{1}{N^{2}-1}\left[1+\frac{k N(N+2)(N-k)}{(k+1)(N-k+1)} e^{-\frac{g^{2} A}{2}(N+1)}\right. \\
\left.+\frac{(N+1)(N-k-1)}{k+1} e^{-\frac{g^{2} A}{2}(N-k)}+\frac{(N+1)(k-1)}{N-k+1} e^{-\frac{g^{2} A}{2} k}\right],
\end{gathered}
$$

$A$ being the area singled out by the loop. The result (1) can be obtained starting from the true $S U(N) / Z_{N}$ theory on the sphere [5], in the decompactification limit or directly on the plane, using the procedure of [6], working with $S U(N)$ and simulating the $k$-sectors with a Wilson loop at infinity in the $k$-fundamental representation.

On the other hand we checked that the very same result can be obtained through a perturbative resummation [5] of the expectation value of an adjoint 
loop enclosed in an asymptotic $k$-fundamental loop on the plane (at least up to $O\left(g^{4}\right)$ ), when the theory is quantized in the light-cone gauge $A_{-}=0$ and on the light front (LF). Hence the perturbative LF formulation seems to capture the exact result, even in presence of a non-trivial topology.

As first pointed out by Witten [7], one can represent the Wilson loop $\mathcal{W}_{k}$ on $S^{2}$ as a sum over instanton contributions. By instanton we mean a non-trivial classical solution of the Yang-Mills equations on $S^{2}$, which takes the form of an Abelian Dirac monopole embedded into the non-abelian gauge group. At variance with the $S U(N)$ or $U(N)$ case, where any of such configurations is characterized by a set of $N$ integers $\left(n_{1}, \ldots, n_{N}\right)$, in the $S U(N) / Z_{N}$ case, by Poisson-resumming on the sphere, instanton numbers are seen to be generalized to rational values by the effect of $k$. The set $(0, \ldots, 0)$, representing the topologically trivial solution, turns out to be reproduced if integration over the group manifold is replaced by integration over the tangent group algebra. In Ref. [2] it was shown, for the case of a Wilson loop in the fundamental representation of the group $U(N)$, that the zero-instanton sector can be obtained by a bon a fide perturbative calculation for the theory quantized in the light-cone gauge by means of equal-time (ET) canonical commutators. In the case of a Wilson loop in the adjoint representation for the $S U(N)$ theory, with $k$-sectors taken into account, an intriguing interplay occurs between instantons and $k$-states and we expect that the truly perturbative physics ignore the existence of the $k$ parameter. In fact the zero-instanton limit does not depend on $k$ and still reproduces the ET computation (in the decompactification limit) without the loop at infinity [5]

$$
\begin{aligned}
& \mathcal{W}_{k}^{(0)}(A)=\frac{1}{N+1}+\frac{N}{\mathcal{Z}(N+1)} \int_{-\infty}^{+\infty} d z_{1} \ldots d z_{N} \exp \left[-\frac{1}{2} \sum_{j=1}^{N} z_{j}^{2}\right] \times \\
& \exp \left[i g\left(z_{1}-z_{2}\right) \sqrt{\frac{A}{2}}\right] \Delta^{2}\left(z_{1}, \ldots, z_{N}\right),
\end{aligned}
$$

where $\mathcal{Z}=\int \mathcal{D} F \exp \left(-\frac{1}{2} \operatorname{Tr} F^{2}\right)$.

Next, we examined the correlation on the sphere between two non-intersecting (nested) loops, one in the adjoint representation and the other in the $k$ fundamental one. Our purpose in so doing was to explore to what extent this procedure reproduces the result we obtained working with a single loop in the adjoint representation on the sphere in a $k$-sector. We found that Witten's conjecture, namely, in our language, that the $S U(N) / Z_{N}$ theory on the plane in a $k$-sector is equivalent to the usual $S U(N)$ theory in presence of a $k$-fundamental Wilson loop at infinity, is indeed verified. However this is true only for the exact solution, and only after decompactification of the sphere. 
We now address the issue of singling the zero-instanton (trivial) sector out for the adjoint loop enclosed in a $k$-fundamental one. The result we obtained [5] shows the following basic features:

(1) for $k \neq 0$, it is different from the single loop case;

(2) although string tensions are independent of $k$, the polynomial coefficients do depend on it.

One should not be too surprised that the instanton structures of the two cases, as long as one remains on the sphere, are completely different. Only for the complete theory on the plane (i.e. full-instanton resummed and then decompactified) the equivalence between $k$-sectors and theories with $k$-fundamental Wilson loops at infinity holds; but there is no reason why this miracle should occur when the two (different) zero-instanton sectors are compared.

We finally checked that the zero-instanton contribution to the expectation value of an adjoint loop enclosed in a $k$-fundamental one on the plane is consistent, at least up to $O\left(g^{4}\right)$, with the perturbative computation where the ET propagator is used.

At this stage, we think we have set a solid ground for the most interesting future development, namely the introduction of dynamical fermions, with a particular focus on the generation and on the properties of a chiral condensate.

\section{References}

[1] S.J. Brodsky, H.C Pauli and S.S. Pinsky, Phys. Rep. 301, 299 (1998) and references therein.

[2] A. Bassetto and L. Griguolo, Phys. Lett. B443, 325 (1998).

[3] E. Witten, Nuovo Cim. 51A, 325 (1979).

[4] G. Grignani, L. Paniak, G.W. Semenoff and P. Sodano, Annals Phys. 260, 275 (1997).

[5] A. Bassetto, L. Griguolo and F. Vian, hep-th/0002093, Annals of Phys. to appear.

[6] L.D. Paniak, G.W. Semenoff and A.R. Zhitnitsky, Nucl. Phys. B487, 191 (1997).

[7] E. Witten, Commun. Math. Phys. 141, 153 (1991) and J. Geom. Phys. 9, 303 (1992). 